\title{
Análisis comparativo de las poblaciones de especies de palmas productoras de "chonta" en los bosques colectivos de la comunidad negra del Alto San Juan, Tadó, Chocó, Colombia
}

\author{
Comparative analysis of populations of species of palms producing \\ "chonta" in collective forests of the black community of Alto San Juan, \\ Tadó, Chocó, Colombia
}

\author{
Jovanny Mosquera-Pino', Mirla Perea-Murillo²
}

\section{Resumen}

En el año 2010, se realizó un estudio de composición y riqueza en las poblaciones de palmas productoras de chonta en un bosque colectivo ubicado en el municipio de Tadó, Chocó, Colombia. Estas palmas han sido cosechadas para extraer trozas o tiras largas de sus tallos denominadas chonta, las cuales vienen sufriendo grandes presiones antrópicas produciendo la disminución de estas poblaciones. Se establecieron muestreos sistemáticos en 191 parcelas de $10 \times 10$ m, equivalentes a 1,91 hectáreas en 6 zonas de muestreos, de las cuales Alto Chato obtuvo 43\%, Manungará 23\%, Angostura 18\%, Carmelo 8\%, Tapón y Corcobado 4\% cada uno de parcelas establecidas, registrando un total de 1480 individuos; de estos, 525 en fustal, 162 latizal y 793 brinzal pertenecientes a palma meme Wettinia quinaria, palma barrigona Iriartea deltoidea, don Pedrito Oneocarpus mapora, murrapo Euterpe precatoria, chappí Euterpe oleraceae y palma zancona Socratea exhorriza, ratonera Astrocaryum jauari Martius y viejita NN. Wettinia quinaria. Se registró la mayor cantidad de individuos (1039) equivalente al 70\% del total identificados, seguido de Iriartea deltoidea y Socratea exhorriza con 8\% cada una, contrario a Euterpe precatoria que solo 7 individuos, siendo la especie de menor abundancia. El estudio demuestra que la presión causada por el aprovechamiento selectivo de palmas productoras de chonta en estas comunidades ha causado que estas poblaciones se encuentren mucho más distantes de los centros poblados; así, Wettinia quinaria es la especie más presionada, sin embargo la cantidad de brinzales (631) y latizal (97) puede garantizar la oferta de chonta en la zona.

Palabras clave: Chonta, Dinámica poblacional, Palma, Riqueza.
Ingeniero Agroforestal, Investigador asociado tipo II del Instituto de Investigaciones Ambientales del Pacífico (IIAP), Quibdó, Chocó.

e-mail: jmosquera@iiap.org.co

2 Ingeniera Agroforestal, Investigadora asociada tipo I del Instituto de Investigaciones Ambientales del Pacífico (IIAP), Quibdó, Chocó.

e-mail: mperea@iiap.org.co

Recibido: 10 de diciembre de 2013

Aceptado: 5 de marzo de 2014

\begin{abstract}
In 2010, a study of composition and richness was conducted in the towns of producing palms chonta in a collective forest located in the town of Tado, Chocó, Colombia. These palms have been harvested to extract logs or long strips from their stems called chonta, which are suffering heavy human pressures leading to the decline of these populations. Systematic sampling were established in 191 plots of $10 \times 10 \mathrm{~m}$, equivalent to 1.91 hectares in 6 areas of sampling, of which Alto Chato got 43\%, Manungará 23\%, 18\% Angostura, Carmelo 8\% cap and Corcobado 4\% each established plots, recording a total of 1480 individuals; of these, 525 fustal, 162 latizal and
\end{abstract}




\section{Bioetnia Volumen 11, 2014}

793 brinzal belonging to Palma Meme Wettinia machinery, Palma Barrigona Iriartea deltoid, Don Pedrito Oneocarpus mapora, murrapo precatoria Euterpe, chappi Euterpe oleraceae and Palma Zancona Socratea exhorriza, Mousetrap astrocaryum janari Martius and Viejita $N N$. Wettinia machinery, recorded the highest number of individuals with 1039 equal to $70 \%$ of the total identified, followed by Iriartea deltoid and Socratea exhorriza with 8\% each, contrary to Euterpe registration precatoria only 7 individuals, being the kind of lower abundance. The study shows that the pressure caused by the selective use of palm producers chonta in these communities has led to such populations are much more distant from population centers; in this order of idea, Wettinia machinery has been the most depressed species, however the amount of saplings (631) and latizal (97) can guarantee the supply of chonta in the área.

Keywords: Chonta, Population dynamics, Palma, Wealth.

\section{Introducción}

En el mundo existe una gran diversidad de palmas representada aproximadamente por 200 géneros y 2.800 especies. Galeano y Bernal (1988). En Colombia hay aproximadamente 47 géneros y 270 especies; para la región del Pacífico se han reportado 106 especies (Bernal y Galeano, 1993); según Gentry (1982) para el departamento del Chocó hay reportado 27 géneros y 81 especies. Los estudios de lineamiento y manejo de las palmas son importante para las comunidades negras, porque en el departamento del Chocó se encuentran aproximadamente $32.2 \%$ de las especies de palmas de Colombia, de las cuales $31.5 \%$ están reportadas en peligro de extinción García et al. (2003); asimismo, el conocimiento de palmas en el Chocó, es resultado de algunas composiciones que se han realizado en diferentes zonas así: García et al. (2003) reportaron 17 géneros y 19 especies; Escobar (2003) determinaron 114 especies de plantas medicinales, mágico-religiosas y alimenticias de las cuales 28 especies son de palmas; Bernal y Ervik (1996) estudiaron la fenología de la inflorescencia y la biología floral, la visita de insectos y el transporte de polen de palma de tagua o marfil vegetal, Phytelephas seemanni; Pino y Valois (2004) encontraron en el corregimiento de Pacurita 70 morfo-especies; asimismo, Ramírez y Galeano (2012) citan que la estructura de la población encontrada tanto en Tutunendo como en Angostura, presentan bajo porcentaje de plántulas con respecto a los juveniles y adultos, lo cual podría sugerir que existen problemas de reclutamiento a nivel general en la comunidad, resultado que reportan los moradores de la zona citando que a finales de la década

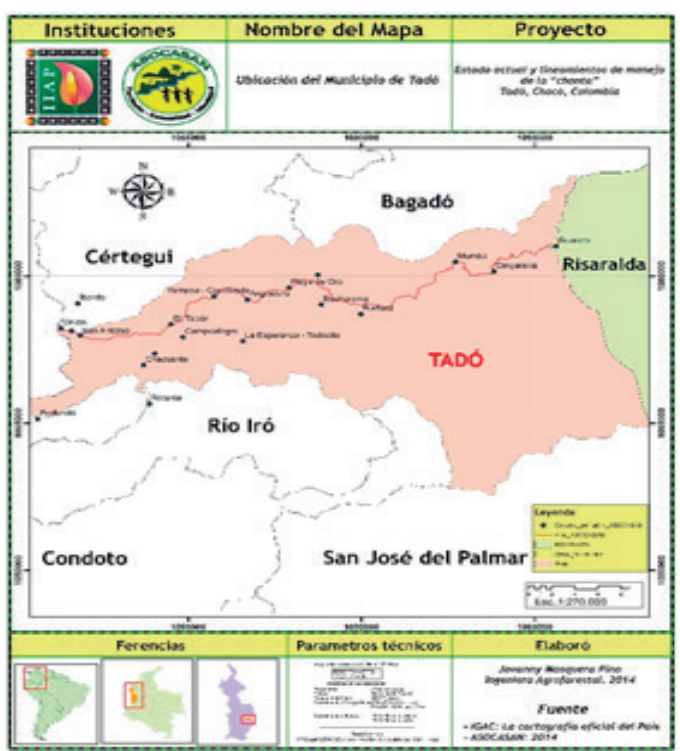

Figura 1. Ubicación del municipio de Tadó.

de 1980, se inició una fuerte presión por cosechar chonta debido a la gran demanda comercial en los departamentos de Risaralda y el Valle del Cauca.

Se conoce como chonta al estado fisiológico del tallo que presentan diferentes especies de palmas, que por sus características de textura y resistencia son cosechados en trozas o tiras largas para la elaboración de galpones, corrales, porquerizas, viviendas, azoteas, entre otros, en las comunidades negras del Alto San Juan. Es así como el Instituto de Investigaciones Ambientales del Pacífico (IIAP), dando respuesta a la preocupación existente en las comunidad negra del Alto San Juan, que ancestralmente han subsistido de la cosecha de chonta y que hoy en día se les dificulta identificar poblaciones cercanas a las comunidades para su extracción, inicia un proceso de socialización, concertación y planificación operativa con los consejos locales que conforman el Consejo Comunitario Mayor del Alto San Juan (ASOCASAN) para determinar la problemática que aqueja a las comunidades, en el marco de la disminución de la producción de chonta y proponer lineamientos de manejo.

Descripción del área de muestreo. El estudio se desarrolló en las comunidades de Alto Chato, Manungará, Tapón, Carmelo, Playa de Oro y Angostura; estas comunidades pertenecen al municipio de Tadó y administradas étnico y territorialmente por el Consejo Comunitario Mayor del Alto San Juan (ASOCASAN) (Figura 1).

El Consejo Comunitario Mayor del Alto San Juan (ASOCASAN), está ubicado en la parte oriental del departamento del Chocó, dentro de la zona del Alto San Juan. La cabecera municipal de Tadó, se encuentra a $66 \mathrm{~km}$ de Quibdó la capital del departamento del Chocó y a 549 km 


\section{Poblaciones de chonta y bosques colectivos. J Mosquera, M Perea}

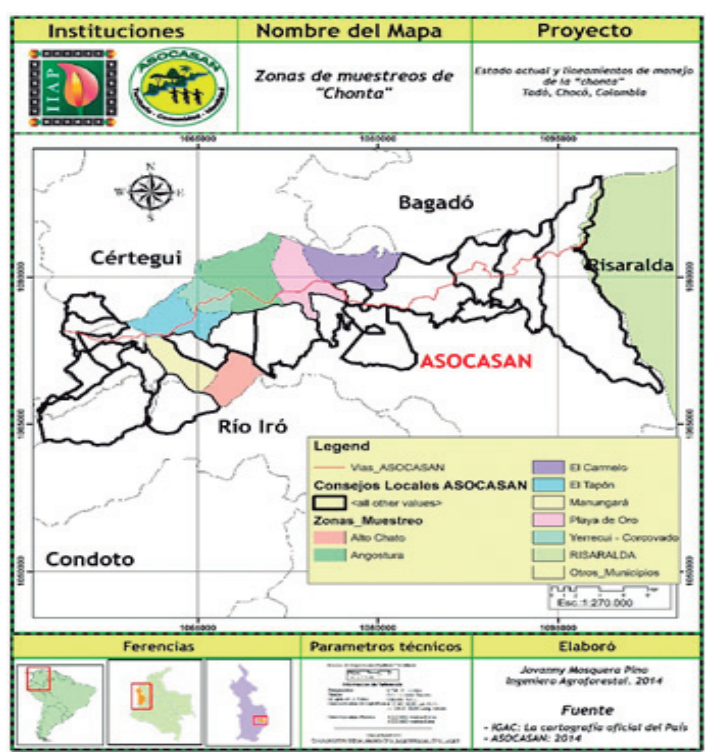

Figura 2. Zonas de muestreo en campo.

de la ciudad de Bogotá. Tiene un área aproximada de 878 $\mathrm{km}^{2}$. Geográficamente se ubica a los $05^{\circ} 15^{\prime} 52.9^{\circ}$ de latitud norte y a $076^{\circ} 33^{\prime} 37.7^{\prime \prime}$ de longitud oeste, con una altura de $90 \mathrm{msnm}$ y posee una temperatura media de $28^{\circ} \mathrm{C}$.

Desde el punto de vista de interés ecosistémico en razón a criterios geomorfométricos, geoestructurales y climatológicos se define el corredor del Alto San Juan como el área de influencia de la parte alta de la cuenca (Tadó, Santa Cecilia-Pueblo Rico) incluyendo las partes altas de las subcuencas de los ríos Iró, Condoto y Tamaná. Los bosques aún persisten en esta zona pese a la intensidad de la actividad minera y forestal.

\section{Método}

Identificación y selección de especies de palmas utilizadas para la extracción dechonta. Pormedio de la realización de mesas de trabajo y conversatorios con investigadores locales, líderes y conocedores en las comunidades de las zonas de muestreos, se listaron las especies de palmas de donde se extrae chonta en el Alto San Juan. Por otra parte, se realizó el diagnóstico sociocultural por medio de entrevistas y encuestas a personas que durante muchos años han subsistido de este subproducto de las palmas.

Establecimiento de parcelas transitorias de muestreos de chonta. El método utilizado para la toma de información primaria fue el de muestreo sistemático diagnóstico, el cual permitió ajustarse a las condiciones específicas del trabajo con la participación de investigadores locales, cinta métrica, GPS, formatos de campo, decámetro, cámara fotográfica y cabuya. Para el análisis de la composición se establecieron 191 parcela de 10 x 10 m equivalente a 1.92 hectáreas, en las siguientes comunidades: Alto Chato (82), Angostura (34), Carmelo (16), en Corcobado (7), Manungará (44), Tapón (8). Una vez identificada las especies se procedió a la selección de un individuo (adulto o juvenil) y trazar a partir de él una cuadrícula de 10 x $10 \mathrm{~m}$. De modo general, se aplicó la metodología propuesta por Hutchinson (1993), con algunas variantes sobre todo en el diseño de las fajas de muestreo; es así que mientras Hutchinson utiliza fajas de 10 $\mathrm{m}$ de ancho por longitud variable, en el presente estudio se consideró la unidad de registro de la cuadrícula de 10 x 10 $\mathrm{m}$, determinando en cada una de ellas el nombre común de la especie de palma, altura total, coordenadas geográficas, circunferencia del fuste, presencia de regeneración natural y estado fitosanitario (Figura 2).

Análisis y sistematización de la información. La determinación del material y el análisis de la información se realizó siguiendo a Rangel (2004) y Mahecha (1997), además de la ayuda de claves taxonómicas, descripciones y las bases de datos como: Herbario Nacional Colombiano (COL), Jardín Botánico de Missouri (MO), New York Botanicals Garden (NY), Real Jardín Botánico (KEW) y especialistas en el conocimiento de la dinámica ecológica de palmas del IIAP. De la misma manera, la compilación y tabulación de los datos se realizó mediante la utilización de base de datos en Office Excel tomando como referente los cuadros y fórmulas establecidas en la guía técnica del Minambiente (2002), las cuales permitieron generar tablas y gráficas dinámicas que facilitaron el análisis de la información. Para la transformación de las coordenadas geográficas a plana se utilizó el programa MAGNA SIRGAS PRO, diseñado por el IGAC (Instituto Geográfico Agustín Codazzi).

Abundancia, frecuencia y riqueza. El análisis estructural de la vegetación correspondiente a especies de palmas utilizada para la extracción de la chonta, se realizó exclusivamente tomando en cuenta el tipo de especies en cada sitio, por lo que sus resultados no deben mirarse de manera absoluta, simplemente constituyen una manera de comparar la situación que en materia de escasez se encuentra en cada sitio. Las respectivas variables se calcularon como se muestra a continuación:

Densidad = Número de individuos/área total del muestreo en hectáreas.

Abundancia relativa $=($ Número de individuos/Número de individuos en el área muestreada) $* 100$.

Frecuencia $=($ Número de unidades de muestreo/Número total de unidades de muestreo) $* 100$

Frecuencia relativa $=($ Frecuencia absoluta/Sumatoria de las frecuencias absolutas) $* 100$

Dominancia relativa $=($ Área basal de cada especie/Área basal total ) *100

Índice de valor de importancia $($ IVI $)=$ Abundancia 
Bioetnia Volumen 11, 2014

Tabla 1. Cantidad de individuos por especie

\begin{tabular}{lrrrr}
\hline \multicolumn{1}{c}{ Especie } & Brinzal & Latizal & Fustal & Total \\
\hline Wettinia quinaria & 631 & 97 & 311 & 1039 \\
Iriartea deltoidea & 50 & 13 & 62 & 125 \\
Socratea exhorriza & 62 & 11 & 43 & 116 \\
Euterpe precatoria & 0 & 2 & 5 & 7 \\
Astrocaryum jauari Martius & 0 & 3 & 7 & 10 \\
NN & 25 & 14 & 43 & 82 \\
Euterpe oleraceae & 21 & 4 & 14 & 39 \\
Oenocarpus minor (O. mapora) Mart & 4 & 18 & 40 & 62 \\
Total & $\mathbf{7 9 3}$ & $\mathbf{1 6 2}$ & $\mathbf{5 2 5}$ & $\mathbf{1 4 8 0}$ \\
\hline
\end{tabular}

Tabla 2. Distribución diamétrica de la chonta

\begin{tabular}{lrrrr}
\hline \multicolumn{1}{c}{ Especies } & $\mathbf{1 0 - 2 0}$ & $\mathbf{2 0 - 3 0}$ & $\mathbf{3 0 - 4 0}$ & Total \\
\hline & 37 & 20 & 2 & 59 \\
Iriartea deltoidea & 11 & 1 & 0 & 12 \\
Euterpe oleraceae & 24 & 6 & 3 & 33 \\
Oenocarpus minor (O. mapora) Mart & 248 & 20 & 2 & 270 \\
Wettinia quinaria & 5 & 0 & 0 & 5 \\
Euterpe precatoria & 6 & 1 & 0 & 7 \\
Astrocaryum jauari Martius & 36 & 5 & 0 & 41 \\
NN & 36 & 4 & 0 & 40 \\
Socratea exhorriza & $\mathbf{4 0 3}$ & $\mathbf{5 7}$ & $\mathbf{7}$ & $\mathbf{4 6 7}$ \\
\hline Total & & & & \\
\hline
\end{tabular}

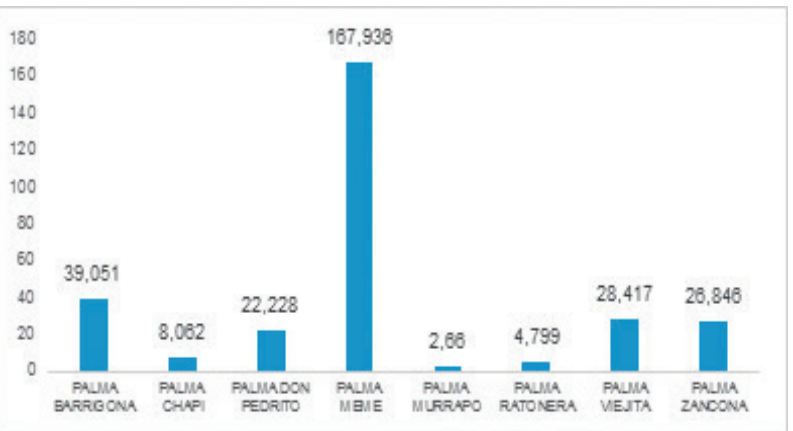

Figura 3. Índice de valor de importancia.

relativa $\%+$ Frecuencia relativa $\%+$ Dominancia relativa $\%$.

Diversidad de especie. Para el análisis de diversidad, se utilizó Estimates versión 9.1.0 determinando la abundancia de cada una de las especies por sitios de muestreos y riqueza específica.

\section{Resultados y discusión}

Se establecieron 190 parcelas, registrando 1.480 in- dividuos en diferente estado de desarrollo: brinzales 793 , latizales 162 y fustales 525 representado en ocho especies de una misma familia (Areacacea). Wettinia quinaria, registró la mayor cantidad de individuos con 1.039 (69\%) del total identificados, seguido de Iriartea deltoidea y Socratea exhorriza con $8 \%$ cada una, contrario a Euterpe precatoria que solo registró 7 individuos, siendo la especie de menor abundancia (Tabla 1).

En relación con el estadío sucesional Wettinia quinaria presenta $80 \%$ de los individuos en brinzal; todo esto se expresa como una excelente condición de su regeneración natural y garantiza la oferta futura de chonta; de igual manera se destaca la inexistencia de regeneración natural de Oneocarpus mapora y Euterpe precatoria; la distribución irregular de Iriartea deltoidea y Socratea exhorriza en su frecuencia y abundancia resulta esperanzadora debido a los individuos en estado juvenil y adulto.

En la Tabla 2 se evidencia que la distribución de los individuos de especies de palma por clases diamétricas muestra de manera contundente la facilidad que tiene la 


\section{Poblaciones de chonta y bosques colectivos. J Mosquera, M Perea}

Tabla 3. Determinación de la diversidad de
especies

\begin{tabular}{lc}
\hline \multicolumn{1}{c}{ Índices } & Resultados \\
\hline Número de individuos (N) & 534 \\
Riqueza de especies (S) & 8 \\
Uniformidad (E) & 0.66569 \\
\hline Índice de Margalef (DMg) & 1.15618 \\
Índice de Simpson (DSp) & 0.37028 \\
Inverso de Simpson (1/DSp) & 2.70063 \\
Índice de Shannon (H') & 1.38426 \\
Varianza de Shannon & 0.00263 \\
Índice de Berger-Parker (d) & 0.57512 \\
\hline Inverso de Berger-Parker (1/d) & 1.73878 \\
Alfa (distrib. logarítmica) & 1.39806 \\
\hline
\end{tabular}

palma memé para que su regeneración natural avance hacia individuos de mayor desarrollo y se convierta en una verdadera alternativa de suministro de materia prima en la zona. También, evidencia la necesidad de desarrollar acciones de salvaguarda y promoción de Euterpe precatoria y Astrocaryum jauari Martius, la cual además, de tener unos escaso número de individuos, no tienen ejemplares en las clases diamétricas superiores; de igual manera, la información consignada a este respecto muestra la necesidad de tomar medidas para la protección de la especie de palma Don Pedrito, que tiene el mayor porcentaje de individuos en las clases diamétricas superiores.

Por otro lado, en el índice de valor de importancia (IVI) se aprecia la gran diferencia en pesos ecológico que tiene la especie de palma memé (167) frente a las otras especies; de igual manera muestra la poca importancia de la palma murrapo, lo que claramente indica el proceso de agotamiento que sufre esta importante especie de palma (Figura 3).

Indice de Margalef (DMg). De acuerdo con los resultados de los cálculos y con las consideraciones que se tienen para el análisis de este índice para el muestreo, se indica que valores inferiores a 2 son considerados como zonas de baja diversidad (resultados de efectos antropogénicos) y valores superiores a 5 son considerados como indicativos de alta biodiversidad (Margalef, 1995).

Índice de Simpson (DSp). A medida que el índice se incrementa, la diversidad decrece. Por ello el Índice de Simpson se presenta habitualmente como una medida de la dominancia. Por tanto, el índice de Simpson sobrevalora las especies más abundantes en detrimento de la riqueza total de especies. Entonces entre más aumente el valor a uno, la diversidad disminuye (Pielou, 1969).

Índice de Shannon $\left(\boldsymbol{H}^{\prime}\right)$. Se expresa con un número positivo, que en la mayoría de los ecosistemas naturales varía entre 1 y 5 . Excepcionalmente puede haber ecosistemas con valores mayores (bosques tropicales, arrecifes de coral) o menores (algunas zonas desérticas). La mayor limitante de este índice es que no tiene en cuenta la distribución de las especies en el espacio.

Índice de Berger-Parker. Es un índice de dominancia que varía entre 0 y 1 , cuanto más se acerca a 1 significa que mayor es la dominancia y menor la diversidad (Magurran, 1988).

\section{Conclusión y discusión}

La palma meme es sin duda la especie de donde más se ha extraído chonta y la que menos probabilidad de agotamiento tiene en este estudio, consecuencia de la alta presencia de regeneración natural la cual crece en el sotobosques y no tiene relación alguna con el porcentaje de supervivencia de las plantas juveniles que a medida que aumentan el crecimiento longitudinal disminuye la población de plantas como consecuencia de la competencia por luz y nutrientes con los individuos en estadío fustal; estas observaciones concuerdan relativamente con Ramírez y Galeano (2012) en el bosque de Angostura que en su mayoría es discontinuo, conformado por parches que soportan las palmas de la localidad, donde posiblemente no son tan eficientes los procesos de dispersión de semillas y establecimiento de nuevos individuos, situación que se evidencia con la abundancia de especies como Wettinia quinaria e Iriartea deltoidea, quienes se encuentran dentro de las 16 especies de palmas más utilizadas por su resistencia según Ledezma y Galeano (2014).

Asimismo, los resultados de abundancia representados en las especies con mayor presencia en fustal debe prestársele mayor atención por su unidad reportada en el área muestreada y su distribución restringida; de igual manera, el mantenimiento de la capacidad de adaptación de las especies por la presión de selección implica obligatoriamente una conservación in situ que presupone una gestión dinámica de los mosaicos del paisaje forestal que deban integrar las eventuales operaciones de silvicultura tendientes al manejo de las especies.

El repoblamiento de las zonas aledañas a los centros poblados (corregimientos y veredas) con palmas se debe realizar identificando zonas de bosques que de acuerdo con las exigencias arriba mencionadas de esta familia, pueden adaptarse bajo estas recomendaciones; esto indica que las palmas en el área de influencia del Consejo Comunitario Mayor del Alto San Juan, son especies de alto valor comercial y de uso doméstico coincidiendo con Ledezma y Galeano (2014) quienes argumentan la importancia económica y cultural de las palmas para los habitantes de la región del 


\section{Bioetnia Volumen 11, 2014}

Pacífico en su artículo sobre el uso de las mismas. Cabe destacar que por los diferentes tipos de usos que antrópicamente se les dio en las comunidades, hoy en día se encuentran un poco dispersa de las mismas, en cantidades abundantes bajo el área de distribución de cada especie, pero que son vulnerables, teniendo en cuenta que comercializadores de chonta, conocen las bondades de las especies y en cualquier momento, de nuevo pueden incursionar en el área y ejercer presión para la comercialización.

Es importante proteger y seleccionar plántulas en semilleros de palmas, con la intensión de garantizar su permanencia y utilizarlos en futuros programas de manejo y recuperación en las especies con mayor probabilidad de agotamiento, porque estas son muy apetecidas por su dureza y utilidad, esto se supone en el marco de un programa de manejo de las especies en escasez que se recomienda como etapa de trabajo siguiente en las comunidades estudiadas; como también, avanzar en la determinación de las características físico químicas de las especies de donde se extrae chonta, con el objeto de generar información relevante tendiente a conocer la dureza, rusticidad, elasticidad y resistencia de cada una de las especies con el ánimo de suministrar dichos resultados a los pobladores y poder determinar el tipo de especie a utilizar a la intemperie o en interiores bajo los diseños de los diferentes sistemas productivos tradicionales.

\section{Literatura citada}

Bernal R, Ervik F. 1996. Floral biology and pollination of the dioecious palms Phytelephas seemannii in Colombia: an adaptation to staphylinid beetles. Biotropica. 28: 682-96.

Escobar DR. 2003. Etnobotánica y comercialización de plantas aromáticas y medicinales en Quibdó, Chocó. Revista Institucional Universidad Tecnológica del Chocó. 8 (19): 12-8.

Galeano-Garcés G y Bernal RG. 1988. Clave sinóptica para los géneros de palmas de Colombia. Mutisia. 69: 1-23.

García F, Ramos YA, Palacios Jdel C, Arroyo JE, Mena A, Gonzáles M (eds.). 2003. Salero: Diversidad biológica de un bosque pluvial tropical (bp-T). Quibdó: Universidad Tecnológica del Chocó.

Gentry AH. 1982. Patterns of neotropical plant species diversity. Evolutionary biology. New York: Plenum Press.

Hutchinson I. 1993. Técnicas silviculturales en bosques tropicales latifoliados. Turrialba: CATIE; $46 \mathrm{pp}$.

Ledezma E, Galeano G. 2014. Usos de las palmas en las tierras bajas del Pacífico colombiano. Caldasia. 36 (1): 71-84.

Mahecha VGE. 1997. Fundamentos y metodología para la identificación de plantas Lener Ltda. Bogotá: Proyecto Ministerio del Medio Ambiente PNUD-GEF Col/92/G31.

Margalef R. 1995. Ecología. Barcelona: Omega.

Magurran A. 1988. Diversidad ecológica y su medición. Barcelona: Ediciones Vedra; pp. 35-9.

Ministerio de Ambiente y Desarrollo Sostenible de Colombia. 2002. Por la cual se establece el salvoconducto nacional para la movilización de productos primarios provenientes de plantaciones forestales. Se modifican las Resoluciones números 0438 y 1029 de 2001 del Ministerio del Medio Ambiente, y se adoptan otras determinaciones; $14 \mathrm{pp}$.

Pielou EC. 1975. Ecological diversity and its measurement. Hoboken: John Wiley \& Sons, Inc.; 165 pp.

Rangel CHOJ. 2004. Colombia diversidad biótica. IV. El Chocó Biogeográfico/ costa pacifica. Bogotá: Universidad Nacional de Colombia.

Ramírez G, Galeano G. 2012. Comunidades de palmas en dos bosques de Chocó, Colombia. Caldasia. 33 (2): 315-29.

Valois H y Pino-Benítez N. 2004. Ethnobotany of four black communities of the municipality of Quibdó, Chocó, Colombia. Lyonia. 7: 59-68. 